\title{
Assessing effective sustainability of fiscal policy within the $\mathrm{G}-7$
}

\author{
Patrick FEVE * \\ Université de Nantes, LEN-C3E and CEPREMAP \\ Pierre-Yves HENIN \\ CEPREMAP and MAD-Université Paris I \\ June 1998 — Revised Version
}

\begin{abstract}
This paper introduces a new unit root test, using "Feedback Covariates", in order to test for sustainability of fiscal policy within the $\mathrm{G}-7$. Non-sustainability is considered as the null hypothesis of both a unit root in the debt process and the nullity of a deficit correction in response to inherited debt. We characterize the distribution of the FADF (Feedback Augmented Dickey-Fuller) statistics under both the null and the near unit root alternative and we compare the FADF statistics with the ones related to single equation approaches. This test is applied to public debt normalized by GDP, rather than by a discount factor, consistently with a definition of effective sustainability, which focusses on policy implications. We are still unable to reject the null hypothesis of non-sustainability for four countries. Nevertheless, our results confirms the potential of our approach in terms of power gains and shows that standard unit root tests leads to accept too often the null hypothesis of non-stationnarity.
\end{abstract}

Keywords : deficit sustainability, policy feedback, unit root tests.

JEL classification : C12, C22, H62

*Corresponding author: Patrick FEVE, CEPREMAP, 142, Rue du Chevaleret, 75013 Paris, France. e-mail: patrick.feve@sc-eco.univ-nantes.fr We thank F. Collard, P. Jolivaldt and F. Langot for fruitful comments. Previous versions of this paper have benefited from discussions at CEEA-Symposium on "Problems of European Monetary Union" 1996 in Frankfurt and EEA conference 1997 in Toulouse. All remaining mistakes are our own. 


\section{Introduction}

Persistent deficits and increases in public debt in most of OECD countries ${ }^{1}$ in the last two decades have motivated a large number of empirical studies devoted to sustainability of fiscal policies. Following the pioneering work of Hamilton and Flavin (1986) for the US economy, many studies have raised this issue, extending the period of analysis or looking at comparative evidence on various OECD countries.

These studies are generally not in accordance on empirical findings. This may be explained by the specificity of national experiences, the definition of sustainability used by econometricians and the empirical strategy adopted. Concerning the last two points, most of sustainability tests are based on the existence of a unit root in the debt process, as incompatible with the satisfaction of the intertemporal budget constraint (see Hamilton and Flavin (1986) or Wilcox (1989)). As such, they suffer from the well known lack of power of unit root tests, especially in small samples. Similar difficulties appear with sustainability tests are performed in a cointegration framework (see Hakkio and Rush (1991), Smith and Zin (1991) and Trehan and Walsh (1988), (1991)) $)^{2}$.

This paper departs from previous empirical studies both in the definition of sustainability and in the econometric approach. We retain a notion of effective sustainability, which imposes the stationarity of public debt expressed in terms of GDP as a (necessary, but not sufficient,) condition for sustainabil-

\footnotetext{
${ }^{1}$ The net public debt as a percentage of nominal GDP for the G-7 has doubled over the past 25 years (see Faruqee, Laxton and Symansky (1997)).

${ }^{2}$ Moreover, as mentioned by Wickens and Uctum (1993) and Bohn (1991), cointegration is not a sufficient condition for sustainability. If primary surplus does not respond positively to higher initial debt, the government policy is not sustainable. This result simply implies that public debt explodes at a rate greater that the discount factor and so the expected discounted debt fails to converge, violating the transversality condition.
} 
ity. This can be viewed as a practical solvency criterion to be opposed to the satisfaction of the intertemporal budget constraint. Concerning the econometric approach, rather than discarding unit root and cointegration tests, we reformulate them in order to increase power. We propose a Feedback Unit Root Test (or Feedback Augmented Dickey Fuller) test statistics, denoted FADF. As a sustainability test, the FADF results from embedding the Bohn (1995) and Wickens and Uctum (1993) auxiliary regression approach within an ADF framework ${ }^{3}$. The null hypothesis corresponds to a joint restriction on the two equations of the system. This leads to test jointly for a unit root in the process of the variables of interest and for the nullity of the feedback mechanism in an auxiliary equation. The paper extends previous results obtained by Fève, Hénin and Jolivaldt (1997), allowing for a drift under the alternative hypothesis of stationarity. The limit distribution of the FADF $t$ statistics is a mixture of the standard normal and Dickey-Fuller distributions, with weights determined by a variance ratio and a correlation parameter of the residuals. Moreover, the paper compares the limit distribution of the FADF statistics with other statistics obtained through a single regression equation. Limit distributions and simulation experiments show the potential of our approach. The key finding is that the FADF statistics performs better in terms of power than usual statistics based on a single regression equation.

The paper is organized as follows. Section 2 introduces definitions and related conditions for effective sustainability. In section 3, we provide and comment the asymptotic distributions of the statistics and we present some simulations experiments. Section 4 is devoted to the empirical implementation on $\mathrm{G}-7$ countries and illustrates the potential of our approach. The last

\footnotetext{
${ }^{3}$ It is worth noting that neither Bohn (1991), nor Wickens and Uctum (1993), cope with these technical difficulties, which result from the fact that the debt is integrated of order one under the null of non-sustainability.
} 
section concludes.

\section{Conditions for effective sustainability}

The standard approach to sustainability considers the satisfaction of the intertemporal budget constraint of the government. We first briefly review the relevant conditions in this case and we focus on additional constraints to be satisfied by any feasible fiscal policy, as required in the alternative effective sustainability approach.

\subsection{From actuarial to effective sustainability}

Usual approaches to sustainability consider the following intertemporal government budget constraint. Given a positive sequence of interest rate $i_{t}$, a stochastic process for the primary surplus $S_{t}$ and assuming no seigniorage, the public debt evolves according to

$$
B_{t}=\left(1+i_{t-1}\right) B_{t-1}-S_{t}
$$

For any positive sequence of interest rates, equation (1) describes an unstable backward dynamics, but may be solved by forward substitution. However, such a forward solution has to satisfy the following transversality condition:

$$
\lim _{s \rightarrow \infty} E_{t} \bar{B}_{t+s}=0
$$

where $E_{t}$ is the conditional expectation and $\bar{B}_{t+s}=\prod_{k=0}^{s}\left(1+i_{t+k-1}\right)^{-1} B_{t+s}$ denotes the discounted future debt for a given sequence of interest rates $\left\{i_{t-1}, \ldots, i_{t+k}, \ldots, i_{t+s}, \ldots\right\}$. This condition implies that the current value of the debt equals the discounted sum of expected primary surpluses, which precludes Ponzi-financing. A necessary requirement for the transversality condition (2) to hold in a stochastic environment is that the discounted debt 
$\bar{B}_{t}$ follows a zero mean stationnary stochastic process. Wilcox (1989) proposes to test directly this condition in order to assess the sustainability of government deficit. This approach inspires a main stream of the empirical research devoted to the topic. ${ }^{4}$

This sustainability condition based on the intertemporal budget constraint of the government thus ensures that government will not play a Ponzi game against private agents, repaying past loans with new loans at an increasing rate. While consistent with a rationality requirement, this is not a practical concept for political feasibility. In a dynamically efficient economy where the interest rate exceeds the growth rate, this criterion does not preclude that the debt and interest charges, taken as ratios over GNP, grow without limits, thus entailing unbounded tax rates and/or primary surpluses ratio. A proper criterion for effective sustainability must account for the boundedness of feasible tax rates. Primary surplus will constitute, as remarked by Kremers (1989), the collateral which guarantees the repayment of public debt. Buiter and Patel (1991) consider actuarial sustainability as the weak criterion and (what we call) effective sustainability as the strong criterion..$^{5}$

A necessary condition for the boundedness of the debt to GNP ratio $b_{t}=B_{t} / Y_{t}$ is that $b_{t}$ follows a stationary process with finite mean $\bar{b}$ and variance $\sigma_{b}$. This allows that the probability for debt or interest charges to exceed any critical value is bounded, although generally not null. This criterion may be expressed as:

$$
\operatorname{Pr}\left(\lim _{t \rightarrow \infty} b_{t}>b_{\text {sup }}\right)<c
$$

where $b_{\text {sup }}$ and $c$ are respectively an arbitrary upper bound for the public

\footnotetext{
${ }^{4}$ For recent developments in the tests of actuarial sustainability, see Quintos (1995).

${ }^{5}$ However; effective sustainability becomes a less restrictive criterion when the GNP growth rate exeeds the interest rate, as in a dynamically inefficient economy.
} 
debt as a percentage of GDP and an arbitrary level of probability. Clearly, when the public debt follows a zero mean stationnary stochastic process, the previous condition can be arbitrarily satisfied. For example, if $b_{t}$ is a small variance iid process, the probability level $c$ may be close to zero. However, stationarity of the public debt is only a necessary condition. Sufficient conditions for effective sustainability impose additional constraints on the mean and the variance of the public debt. Conversely, when public debt is integrated of order one, the probability for $b_{t}$ to exceed $b_{\text {sup }}$ is always equal to 0.5 , whatever the value of the upper bound is. This is because in the case of a random walk, public debt behaves ${ }^{6}$ as $\sqrt{T} \sigma_{b} \varepsilon_{b, T}$ as $T \rightarrow \infty$.

\subsection{Debt and surplus dynamics in a growing economy}

In order to associate operational restrictions to the effective sustainability conditions, we have to set up the relations between debt and surplus dynamics in a growing economy. The dynamics of the debt ratio is now given by

$$
b_{t}=\left(1+n_{t-1}\right)^{-1}\left(1+i_{t-1}\right) b_{t-1}-s_{t}
$$

where $s_{t}=S_{t} / Y_{t}$ is the primary surplus ratio and $n_{t}$ is the growth rate of GDP. The dynamics of effective public debt depends now on the value of the ratio of the interest factor over the growth factor. Without restriction on the sequences of interest and growth rates, debt dynamics may be reformulated as

$$
b_{t}=b_{t-1}-g c s_{t}
$$

when $g c s_{t}$ is the "growth corrected surplus" ratio, inclusive of interest, defined as

$$
g c s_{t}=s_{t}+\left(i_{t-1}-n_{t-1}\right) b_{t-1}
$$

\footnotetext{
${ }^{6}$ For this result, we must only impose than $\lim _{T \rightarrow \infty} b_{\text {sup }} / \sqrt{T}=0$. Obviously, a constant value of this upper bound leads to the same conclusion.
} 
This formulation has two appealing features. First, it does not impose restrictions on the dynamic properties of the real interest rate. Second, it allows to test for the stationarity of the public debt ratio, which can be viewed a necessary but not sufficient condition for effective sustainability. ${ }^{7}$ Clearly, the debt ratio would follow an integrated process except in two cases, where proper restrictions are imposed on the (corrected) deficit process:

i) the growth corrected surplus is uncorrelated with $b_{t-1}$, but over differentiated $(\sim I(-1))$. This case appears neither likely to occur, nor interesting to comment.

ii) the growth corrected surplus may be expressed as a function of $b_{t-1}$

$$
g c s_{t}=\alpha+\beta b_{t-1}+u_{t}
$$

with the restriction $\beta>0$.

As far as the identity $\Delta b_{t} \equiv-g c s_{t}$ holds each period, the reduced form of equations (4)-(6) define a single equation where the effective public debt follows an autoregressive process. Such an identity is not warranted to hold in any practical situation and we consider an alternative formulation

$$
\Delta b_{t}=-g c s_{t}+\varepsilon_{t}
$$

with $\varepsilon_{t}$ a stationary disturbance with a possible non-zero mean, denoted $\delta$. A first rationale for introducing this disturbance term in an empirical study relies on accounting discrepancies between the flow accounting used to measure gcs $_{t}$ and the stock accounting underlying measures of public debt. Holzmann, Herve and Demmel (1996) convincingly argue on the importance

\footnotetext{
${ }^{7}$ This also corresponds to institutional arrangements, such as the Maastricht criteria for European countries.
} 
of this measurement error including the following components: the valuation differences between nominal and market values, the valuation effect of government net liabilities of exchange rate changes, the net increase of other government assets (industrial participation, trade credit, ....). According to Holzmann and alii (1996), this residual accounts roughly for one third of the change in the debt. As we will demonstrate in the following section, a second rationale relies on the efficiency gains in statistical inference. Rather than complicate the econometric exercise, this measurement error allows to fully combine the joint dynamics of the debt ratio and the growth corrected surplus ratio.

\section{A "feedback augmented" test for sustain- ability}

The stationarity of the debt ratio $b_{t}$, as a necessary condition for effective sustainability may be assessed using an usual unit root test. As it is well known, such a test lacks of power, especially against near unit root alternative (see Schwert (1989), or Campbell and Perron (1991)). As debt is a stock variable, resulting from an accumulation process, it is likely to be very persistent, with a (larger) autoregressive root close to one even in case of a sustainable fiscal policy. An alternative strategy is to test for the stationarity of the gross surplus ratio (inclusive of interest) $g s_{t}$, which can be shown to be a necessary and sufficient condition for stationarity of $b_{t}$. However, this alternative approach suffers from ignoring relevant information by discarding the empirical process of the main variable of interest $b_{t}$, from empirical inference. We first present our regression framework and the asymptotic distribution of the statistics under the null hypothesis on non sustainability. Simulation experiments illustrate the potential of our approach in terms of power gain. 


\subsection{The regression framework}

A more efficient strategy is to consider the surplus equation (6) as an auxiliary equation providing useful information on a feedback mechanism contributing to debt stabilization. Substituting (7) in (6), we have

$$
b_{t}=\delta-\alpha+(1-\beta) b_{t-1}+\varepsilon_{t}-u_{t}
$$

This equation constitutes a constrained reduced form of which the standard Dickey Fuller equation is the unconstrained counterpart. Considering (6) and (8) as a system based equations allows to take advantage from cross restrictions. Denoting $1-\beta$ by $\rho$, we have ${ }^{8}$

$$
\begin{aligned}
\Delta y_{t} & =\delta-\alpha+(\rho-1) y_{t-1}+\varepsilon_{t}-u_{t} \\
x_{t} & =\alpha+(1-\rho) y_{t-1}+u_{t}
\end{aligned}
$$

where $y_{t}=b_{t}$ and $x_{t}=g c s_{t}$. The null hypothesis to be tested is $\rho=1$ and $\delta=\alpha=0$ in equations (9)-(10), i.e. the joint hypothesis of a unit root in the debt ratio process and of independence of the growth corrected surplus with respect to debt ratio. This test was introduced in Fève and Hénin (1996) as a "feedback augmented" unit root test and a comprehensive characterization of the associated FADF statistics is provided in Fève, Hénin and Jolivaldt $(1998)^{9}$. This paper extends these previous results, allowing for a drift under the alternative hypothesis of stationarity. It also compares the asymptotic properties of the FADF statistics with the ones of single regression approaches, either the usual $\mathrm{ADF}$ t-statistics from equation (9),

\footnotetext{
${ }^{8}$ Elliott and Stock (1994) and Cavanagh, Elliott and Stock (1995) consider a somewhat similar system based equations, but with no cross equations restriction on the parameter of interest and a simpler cross correlation structure between the disturbances.

${ }^{9}$ In this previous paper, the FADF statistics is thus compared to the Covariates-OLS (Phillips and Hansen (1990)) and Covariates-ADF (Hansen (1995)) statistics. The main results is that COLS and CADF statistics may be biased and have very low power.
} 
either the $t$-statistics associated to the AR-OLS (auxiliary regression OLS) estimate in equation (10).

The sequence $\left\{\varepsilon_{t}, u_{t}\right\}$ is assumed to be iid and orthogonal to the regressor. We do not exclude contemporaneous correlation between the two error terms. The covariance matrix of $\left\{\varepsilon_{t}, u_{t}\right\}$ is given by

$$
\Sigma_{1}=\left(\begin{array}{cc}
\sigma_{\varepsilon}^{2} & \sigma_{\varepsilon u} \\
\sigma_{\varepsilon u} & \sigma_{u}^{2}
\end{array}\right) \equiv \sigma_{u}^{2}\left(\begin{array}{cc}
\eta^{2} & \tau \eta \\
\tau \eta & 1
\end{array}\right)
$$

where $\tau=\operatorname{Cov}\left(\varepsilon_{t}, u_{t}\right)\left(\sigma_{\varepsilon} \sigma_{u}\right)^{-1}$ is the correlation parameter between $\varepsilon_{t}$ and $u_{t}$, which satisfies $|\tau|<1$ and $\eta^{2}=\sigma_{\varepsilon}^{2} \sigma_{u}^{-2}$ is the variance ratio. In what follows, we can express the limit distribution of the statistics in terms of these two parameters. As mentioned previously, we consider the regression framework

$$
\begin{aligned}
\Delta y_{t} & =(\widehat{\rho}-1) y_{t-1}+\widehat{\delta}-\widehat{\alpha}+\widehat{\varepsilon}_{t}-\widehat{u}_{t} \\
x_{t} & =(1-\widehat{\rho}) y_{t-1}+\widehat{\alpha}+\widehat{u}_{t}
\end{aligned}
$$

The natural test statistics for the hypothesis of unit root in models (11)(12) is the t-statistics

$$
t_{(.)}^{\mu}=\frac{\widehat{\rho}-1}{s(\widehat{\rho}-1)}
$$

where $s(\widehat{\rho}-1)$ is the standard error for $\widehat{\rho}-1$ and the subscript $()=$. $\{(F A D F),(A D F),(A R-O L S)\}$ according to which of the following cases is considred. Case (a) will correspond to the ML estimates of $\rho, \alpha$ and $\delta$ from equations (11)-(12). The associated FADF $t$-statistics is denoted $t_{(F A D F)}^{\mu}$. Case (b) will ignore cross equations restriction and considers only the equation (11). It corresponds to usual univariate unit root test. The associated ADF t-statistics is denoted $t_{(A D F)}^{\mu}$. Finally, Case (c) will correspond to OLS estimates of $\rho$ and $\alpha$ from the auxiliary equation (12). The associated OLS t-statistics is denoted $t_{(A R-O L S)}^{\mu}$. 


\subsection{Asymptotic Results}

The asymptotics provided here are related to a prototypical model, under a set of strong assumptions. This is partly because it makes the limit distributions easier to obtain. The asymptotic theory is based on "local-to-unity" asymptotic, following Phillips (1987b). The model has a unit root when $\rho=1$. This constitutes the null hypothesis of interest. We consider here local departure from the null hypothesis with setting

$$
\rho=1-\frac{c}{T}
$$

The null hypothesis holds when $c=0$ and holds "locally" as $T \rightarrow \infty$ for $c \neq 0$. The asymptotic theory for near-integrated processes uses the diffusion representation, with the associated functional $B_{1}^{c *}(r)=B_{1}^{c}(r)-\int_{0}^{1} B_{1}^{c}(s) d s$. we obtain the following asymptotic distribution for the three t-statistics:

Theorem 1 Under the local alternative $c \neq 0$, as $T \rightarrow \infty$,

$$
\begin{aligned}
& (a 1) t_{(F A D F)}^{\mu} \Longrightarrow-c \xi^{-1} A_{1}^{c}+\xi A_{2}^{c}++\left(1-\xi^{2}\right)^{1 / 2} \mathcal{N}(0,1) \\
& (b 1) t_{(A D F)}^{\mu} \Longrightarrow-c A_{1}^{c}+A_{2}^{c} \\
& (c 1) t_{(A R-O L S)}^{\mu} \Rightarrow-c\left(1-\tau^{2}\right)^{1 / 2} \xi^{-1} A_{1}^{c}+\varkappa A_{2}^{c}+\left(1-\varkappa^{2}\right)^{1 / 2} \mathcal{N}(0,1)
\end{aligned}
$$

Under the null hypothesis $c=0$, as $T \rightarrow \infty$,

(a2) $t_{(F A D F)}^{\mu} \Longrightarrow \xi A_{2}+\left(1-\xi^{2}\right)^{1 / 2} \mathcal{N}(0,1)$

(b2) $t_{(A D F)}^{\mu} \Longrightarrow A_{2}$

(c2) $t_{(A R-O L S)}^{\mu} \Rightarrow \varkappa A_{2}^{c}+\left(1-\varkappa^{2}\right)^{1 / 2} \mathcal{N}(0,1)$

where $\xi=(1-\tau)^{1 / 2}\left(1+\eta^{2}-2 \eta \tau\right)^{-1 / 2}, \varkappa=\frac{1-\eta \tau}{\left(1-\tau^{2}\right)^{1 / 2}} \xi, A_{1}^{c}=\left\{\int_{0}^{1}\left\{B_{1}^{c *}(r)\right\}^{2} d r\right\}^{1 / 2}$

and $A_{2}^{c}=\int_{0}^{1} B_{1}^{c *}(r) d B_{1}(r) / A_{1}^{c}$

Parts (a1) and (a2) of Theorem 1 are just an extension of the results of Feve, Henin and Jolivaldt (1997) to the case of a drift term in the regression equations under the null of unit root. Parts (b1) and (b2) concern the limit 
distributions of near integrated processes(see Phillips (1987)). Finally, parts (c1) and (c2) display the second-order biases of the OLS single regression estimate. ${ }^{10}$ This case is related to the one considered in Cavanagh, Elliot and Stock (1995), where the weighting parameter reduces to the correlation parameter between the disturbances. ${ }^{11}$ These authors also find that an analogue to the AR-OLS t-statistics contains an ADF component as long as the disturbances of the system-based equations are correlated.

Parts (a1) and (a2) of Theorem 1 provide the asymptotic distribution of the FADF $t$-statistics under the local-to-unity alternative and under the null hypothesis. The asymptotic distribution is a mixture of the Dickey-Fuller and the standard normal distributions. The combination is not linear and also the sum of the weights is not equal to one. $\xi$ appears as a non-linear function of $\eta$ and of $\tau$ and it appears difficult to obtain general interpretation. However, we observe that, as $\eta$ grows, the importance of the Dickey-Fuller statistics globally decreases. Moreover, as $\tau$ grows from -1 to 1 , the limit distribution moves from a Dickey-Fuller distribution to a normal one. The limit distribution of the FADF t-statistics shows that gains in power are associated to a normal limit distribution. This is because a small value of $\xi$ implies a departure from the Dickey-Fuller type distribution and, for $c$ positive, the limit distribution takes large negative value.

Parts (b1) and (b2) of Theorem 1 correspond to the limit distribution of the ADF t-statistics (see Phillips (1987a) and (1987b)). The limit distribution is free of the nuisance parameter $\xi$. The limit distribution of $t_{(F A D F)}^{\mu}$

\footnotetext{
${ }^{10}$ This case exhibits the main limitations involved by the single auxiliary regression approach, i.e. it does not allow to identify the nuisance parameter $\varkappa$.

${ }^{11}$ Cavanagh, Elliot and Stock (1995) consider a recursive representation, where the parameter of interest in the system based equations is not structurally related. They also normalize both disturbances to one in the data generating process and thus eliminate an extra nuisance parameter.
} 
and $t_{(A D F)}^{\mu}$ are the same only in the particular case $\xi=1$ which corresponds to the constraint $\eta=\tau$. This implies that power of the ADF t-statistics is the same of the FADF t-statistics only in this particular case. Generally, one may expect loss in power for the ADF statistics, compared to the FADF one.

Parts (c1) and (c2) of Theorem 1 provide the limit distribution of the AR-OLS t-statistics from the second equation considered in isolation. ${ }^{12}$ The asymptotic distribution is a pseudo-convex mixture of the Dickey-Fuller and the standard normal distributions. The limit distribution of $t_{(F A D F)}^{\mu}$ and $t_{(A R-O L S)}^{\mu}$ are the same only in the particular case $\tau=0$. When $\eta=\tau$, the t-statistics may have less power than the FADF and ADF statistics. In this case, $\xi=1$ and for absolute values of $\tau$ near one, the limit distribution of the statistics is always a normal one, whatever the values of $c$. A main shortcoming of tests based on the auxiliary regression considered in isolation is that they rest on a statistics depending on a nuisance parameter $\varkappa$, not identified within this single equation approach.

The cases (b) and (c) can be deduced from the estimates of $\rho$ in the general case (a). The ML estimate of $\rho$ is given by

$$
\begin{aligned}
\widehat{\rho}_{F A D F} & =1+\frac{\tau}{\eta} \frac{\sum y_{t-1} \Delta y_{t}}{\sum y_{t-1}^{2}}+\frac{\tau-\eta}{\eta} \frac{\sum y_{t-1} x_{t}}{\sum y_{t-1}^{2}} \\
& =1+\frac{\tau}{\eta}\left(\widehat{\rho}_{A D F}-1\right)+\frac{\eta-\tau}{\eta}\left(\widehat{\rho}_{A R-O L S}-1\right) \\
& =\frac{\tau}{\eta} \widehat{\rho}_{A D F}+\frac{\eta-\tau}{\eta} \widehat{\rho}_{A R-O L S}
\end{aligned}
$$

The ML estimate of $\rho$ is a linear combination of the univariate autoregressive estimate and the auxiliary regression estimate. If $\eta=\tau$, the second term vanishes and we obtain the limit distribution of univariate unit root test. When $\tau=0$, the estimated value of $\rho$ corresponds to the single auxiliary

\footnotetext{
${ }^{12}$ These results are relevant to characterize the distribution of OLS estimates used by Bohn (1991) and underline the difficulties to determine a proper test following his proposal.
} 
regression approach without covariates.

\subsection{Simulations experiments}

In order to illustrate the previous asymptotics, we present some simulation experiments. These experiments may be considered as representative of our sample data: the sample size is small $(T=60)$, the values of $\eta$ and $\tau$ corresponds to three typical configurations obtained from the data and the local departures from the unit root are calibrated on the basis of the estimated values of the autoregressive parameters

We first present the empirical pdf of the autoregressive parameter for each approach in three specific configurations of the DGP, i.e. for different pairs $\{\eta, \tau\}$. FADF, ADF and AR-OLS estimates correspond to the solid line, the dashdotted line and the dotted line, respectively. Empirical pdf are obtained from 2500 samples of size 60 with iid Gaussian innovations, under the null hypothesis of unit root and the nullity of the feedback effect.

In figure 1 , we report the empirical pdf for $\eta=1$ and $\tau=0.5$. The estimated value of $\rho$ in the FADF case is a convex mixture of the ADF and AR-OLS ones, with a weight equal to 0.5. Figure 1 illustrates this result and show that in this case none of the approaches provide similar value of $\rho$. Moreover, the empirical distribution in the FADF case appears more concentrated than the one in the ADF case.

In figure 2, we report the empirical pdf of $\rho$ in the particular case where the variance ratio and the correlation parameter are equal $(\eta=0.6$ and $\tau=0.6$.). From theorem 1 , we know that the FADF statistics admit the same limit distribution than the ADF statistics. This result is confirmed by figure 2 , where the two empirical pdf are very closed. Conversely, the empirical pdf of $\rho$ obtained from AR-OLS differs significantly and the distribution of the 


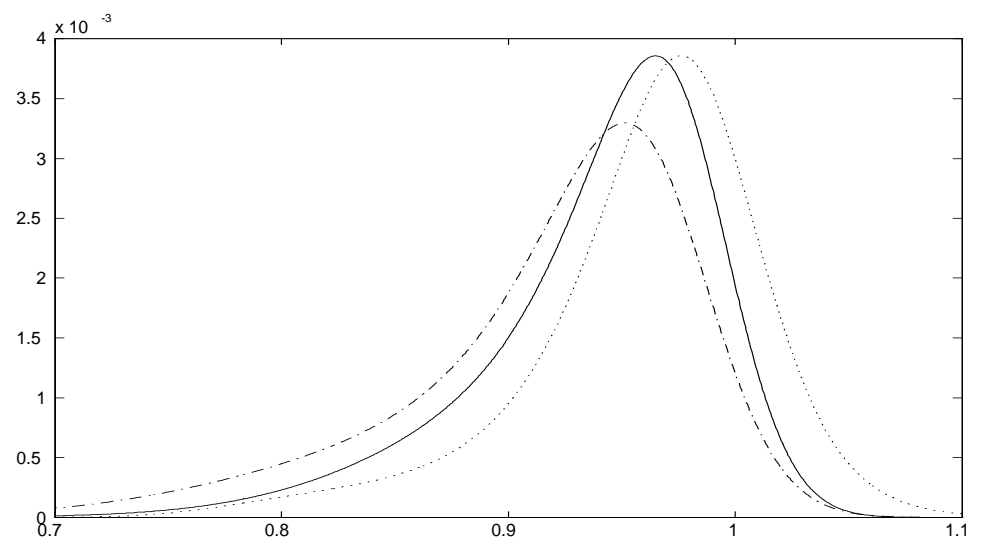

Figure 1: Empirical pdf of $\rho$ (case 1: $\eta=1, \tau=0.5)$. FADF, ADF and AR-OLS estimates correspond to the solid line, the dashdotted line and the dotted line, respectively.

autoregressive parameter appears less concentrated. Finally, in figure 3, we report the empirical pdf of $\rho$ in the case where the variance ratio takes values less than the ones of the correlation parameter $(\eta=0.5$ and $\tau=0.7$.). The two empirical pdf of FADF and ADF estimates appear quite close and they differ significantly from the AR-OLS estimate. Moreover, estimated values of $\rho$ in the $\mathrm{ADF}$ and $\mathrm{AR}-\mathrm{OLS}$ cases appear less concentrated than the ones obtained from an FADF regression.

Table 1 reports the t-statistics and their power. The mean value and standard-error of $\rho$ first indicates consistency and efficiency of the various approaches. Reported values may be used to compare the empirical pdf in figures 1,2 and 3.

The first experiment considers $\eta=1, \tau=0.5$. In this case, the FADF and AR-OLS approaches provide better results in terms of biases reduction, efficiency and power than the usual ADF approach. The second experiment consider the pair $\eta=0.6, \tau=0.6$. From Theorem 1 and the related 


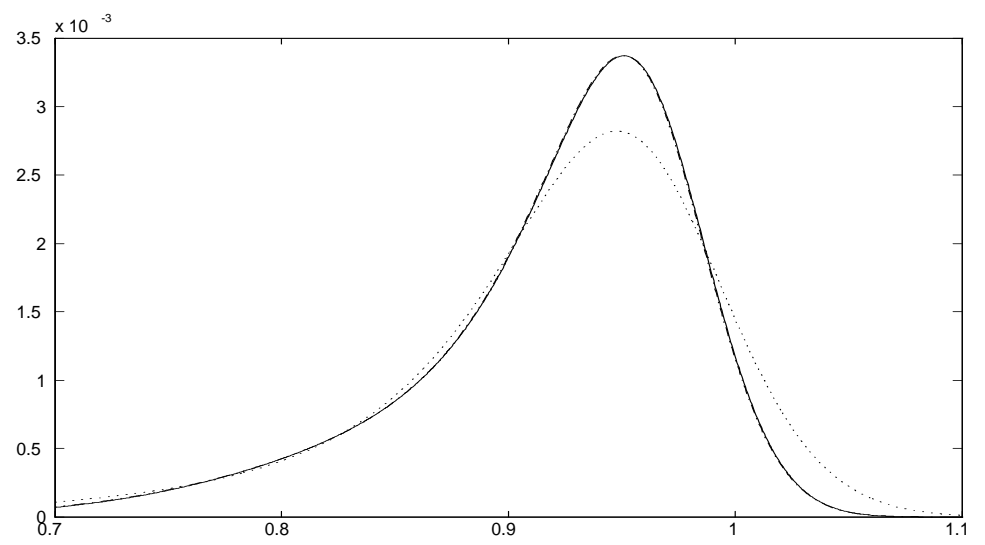

Figure 2: Empirical pdf of $\rho$ (case 2: $\eta=0.6, \tau=0.6)$. FADF, ADF and AROLS estimates correspond to the solid line, the dashdotted line and the dotted line, respectively.

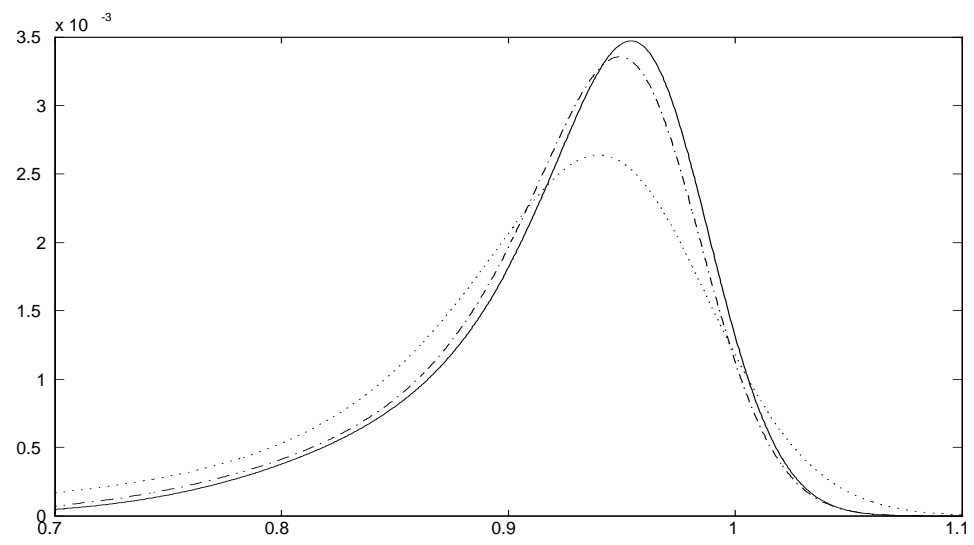

Figure 3: Empirical pdf of $\rho$ (case $3: \eta=0.5, \tau=0.7)$. FADF, ADF and AROLS estimates correspond to the solid line, the dashdotted line and the dotted line, respectively. 
Table 1: Critical values and power of the statistics

\begin{tabular}{|c|c|c|c|c|c|c|c|c|c|}
\hline & FADF & $\mathrm{ADF}$ & $\mathrm{AR}-\mathrm{OLS}$ & FADF & $\mathrm{ADF}$ & AR-OLS & FADF & $\mathrm{ADF}$ & AR-OLS \\
\hline & \multicolumn{3}{|c|}{$\eta=1, \tau=0.5$} & \multicolumn{3}{|c|}{$\eta=0.6, \tau=0.6$} & \multicolumn{3}{|c|}{$\eta=0.5, \tau=0.7$} \\
\hline mean & 0.938 & 0.917 & 0.960 & 0.917 & 0.917 & 0.919 & 0.922 & 0.917 & 0.903 \\
\hline s.e. & 0.055 & 0.067 & 0.054 & 0.067 & 0.067 & 0.075 & 0.064 & 0.067 & 0.087 \\
\hline \multicolumn{10}{|c|}{ critical values } \\
\hline & \multicolumn{3}{|c|}{$t^{\mu}$} & \multicolumn{3}{|c|}{$t^{\mu}$} & \multicolumn{3}{|c|}{$t^{\mu}$} \\
\hline $1 \%$ & -3.41 & -3.52 & -3.14 & -3.54 & -3.52 & -3.25 & -3.58 & -3.52 & -3.39 \\
\hline $5 \%$ & -2.82 & -2.91 & -2.33 & -2.93 & -2.91 & -2.67 & -2.85 & -2.91 & -2.75 \\
\hline $10 \%$ & -2.48 & -2.59 & -1.96 & -2.64 & -2.59 & -2.30 & -2.60 & -2.59 & -2.38 \\
\hline \multicolumn{10}{|c|}{ Power in $\%$ at $5 \%$} \\
\hline$\rho$ & \multicolumn{3}{|c|}{$t^{\mu}$} & \multicolumn{3}{|c|}{$t^{\mu}$} & \multicolumn{3}{|c|}{$t^{\mu}$} \\
\hline 0.99 & 6.1 & 5.2 & 6.4 & 5.9 & 5.2 & 4.6 & 7.0 & 5.2 & 4.5 \\
\hline 0.95 & 12.2 & 9.2 & 14.1 & 9.8 & 9.2 & 7.8 & 11.7 & 9.2 & 6.2 \\
\hline 0.90 & 26.3 & 16.3 & 27.2 & 18.4 & 16.3 & 13.5 & 23.4 & 16.3 & 9.8 \\
\hline
\end{tabular}

discussion, we have the same distribution for the FADF and ADF statistics. Small departures appear. This is because, we have to obtain a consistent estimate the covariance matrix of residuals. Nevertheless, small differences in the critical values reveal small size distortions. For example, the $5 \%$ critical value of the $\mathrm{ADF}$ t-statistics corresponds to the $5.2 \%$ critical value of the FADF one. Moreover, a simple P-Plot curve shows that the P-values of the FADF are close to the straight line and indicates a good agreement between the two statistics. The power of the AR-OLS statistics is lower than the FADF and ADF ones. These results are reinforced in the last case $\eta=0.5$ , $\tau=0.7$. This case illustrates well the gains in power when the FADF statistics is used, instead the ADF or AR-OLS ones. The FADF statistics dominates the other statistics in terms of power, even if for a small sample size, the gain is power is not very large. 


\section{Effective sustainability among the G-7 mem- bers}

We now look at the application of our empirical strategy to the quantitative evaluation of the effective sustainability within the $G-7$. Data are taken from the "OECD Economic Outlooks" Dataset. The frequency is semi-annual and the sample period covers for the majority of countries the last three decades. For each country, we use the net public debt (code GNFL) and the net lending as measure of global surplus (code NLG). The public debt and global surplus are both expressed as a fraction of nominal GDP.

The sample size is quite small because it lies between 50 (France, Japan) and 75 (US, Germany, Canada). Nevertheless, our simulation experiments have shown that the FADF statistics may have some power in small sample, contrary to the ADF statistics or AR-OLS statistics associated to a single regression approach. Our asymptotic results are obtained under the strong assumption of iid errors terms. Such an assumption cannot be maintained in practice, because first difference of effective public debt and growth corrected global surplus display both significant temporal dependance. Nevertheless, as shown in Feve, Henin and Jolivaldt (1997), the limit distributions of the $t$ statistics remains valid in presence of serially correlated errors if an adequate specification is adopted, i.e. if we introduce in our system based equation lagged values of the dependent variables.

We first select under the null hypothesis $\rho=1$ the order of the serial correlation from the following representation:

$$
\begin{aligned}
b_{t} & =\delta-\alpha+\rho b_{t-1}+\sum_{i=1}^{p} \theta_{11, i} \Delta b_{t-i}+\sum_{i=1}^{p} \theta_{12, i} g c s_{t-i}+\varepsilon_{t}-u_{t} \\
g c s_{t} & =\alpha+(1-\rho) b_{t-1}+\sum_{i=1}^{p} \theta_{21, i} \Delta b_{t-i}+\sum_{i=1}^{p} \theta_{22, i} g c s_{t-i}+u_{t}
\end{aligned}
$$


Using AIC and BIC criteria, we select two lags $(p=2)$ for each country. Moreover, the parameters $\theta_{12, i}$ and $\theta_{21, i}, \forall i=1,2$, are not significantly different from zero. Specification tests on the selected model indicate that we cannot reject the hypothesis that the errors terms are white noise.

Table 2: Estimation Results

\begin{tabular}{|c|c|c|c|c|}
\hline & & FADF & $\overline{\mathrm{ADF}}$ & OLS \\
\hline US & $\widehat{\rho}$ & 0.966 & 0.968 & 0.969 \\
\hline$\eta=0.61$ & $\operatorname{se}(\widehat{\rho})$ & 0.012 & 0.012 & 0.016 \\
\hline \multirow[t]{2}{*}{$\tau=0.67$} & t-stat & -2.87 & -2.62 & -1.93 \\
\hline & P-val. & $5.0 \%$ & $8.5 \%$ & $21.0 \%$ \\
\hline UK & $\widehat{\rho}$ & 0.964 & 0.973 & 0.953 \\
\hline$\eta=0.87$ & $\operatorname{se}(\widehat{\rho})$ & 0.012 & 0.014 & 0.016 \\
\hline \multirow[t]{2}{*}{$\tau=0.39$} & $t$-stat & -3.00 & -1.93 & -3.00 \\
\hline & P-val. & $3.5 \%$ & $30.5 \%$ & $1.7 \%$ \\
\hline Germany & $\widehat{\rho}$ & 1.004 & 1.005 & 1.003 \\
\hline$\eta=1.50$ & $\operatorname{se}(\widehat{\rho})$ & 0.006 & 0.009 & 0.007 \\
\hline \multirow[t]{2}{*}{$\tau=0.48$} & t-stat & 0.69 & 0.54 & 0.45 \\
\hline & P-val. & $95.6 \%$ & $98.2 \%$ & $78.8 \%$ \\
\hline France & $\widehat{\rho}$ & 1.027 & 1.029 & 1.013 \\
\hline$\eta=1.30$ & $\operatorname{se}(\widehat{\rho})$ & 0.013 & 0.019 & 0.017 \\
\hline \multirow[t]{2}{*}{$\tau=0.44$} & $t$-stat & 2.04 & 1.53 & 0.77 \\
\hline & P-val. & $99.7 \%$ & $99.7 \%$ & $89.7 \%$ \\
\hline Italy & $\widehat{\rho}$ & 1.002 & 1.004 & 1.011 \\
\hline$\eta=0.57$ & $\operatorname{se}(\widehat{\rho})$ & 0.006 & 0.006 & 0.008 \\
\hline \multirow[t]{2}{*}{$\tau=0.38$} & $t$-stat & 0.33 & 0.59 & 1.44 \\
\hline & P-val. & $97.1 \%$ & $98.8 \%$ & $99.5 \%$ \\
\hline Canada & $\widehat{\rho}$ & 0.999 & 0.997 & 0.990 \\
\hline$\eta=0.76$ & $\operatorname{se}(\widehat{\rho})$ & 0.006 & 0.006 & 0.011 \\
\hline \multirow[t]{2}{*}{$\tau=0.77$} & t-stat & -0.02 & -0.51 & -0.99 \\
\hline & P-val. & $94.9 \%$ & $94.8 \%$ & $47.9 \%$ \\
\hline Japan & $\widehat{\rho}$ & 0.977 & 0.978 & 0.982 \\
\hline$\eta=0.85$ & $\operatorname{se}(\widehat{\rho})$ & 0.005 & 0.006 & 0.008 \\
\hline \multirow[t]{2}{*}{$\tau=0.60$} & t-stat & -4.47 & -3.77 & -2.31 \\
\hline & P-val. & $0.2 \%$ & $0.5 \%$ & $6.5 \%$ \\
\hline
\end{tabular}

Given our selected model under the null hypothesis of unit root, we may compute the covariance matrix of residuals. This provides consistent es- 
timates for the parameters $\eta$ and $\tau$. Estimation results with $\mathrm{G}-7$ data indicate three types of configurations of the covariance matrix. A first configuration corresponds to the case where $\eta>\tau$, a second where $\eta \approx \tau$, while the third corresponds to $\eta<\tau$. As Theorem 1 shows, one may expect that FADF and AR-OLS t-statistics have better power properties than the ADF one in the first case. Conversely, in the third case, the FADF and ADF t-statistics have better power properties than the AR-OLS one.

We report in table 2 the parameter estimates and their associated $t$ statistics . Given the particular values of $\eta$ and $\tau$, the critical values of the t-statistics are obtained through simulations for each country. Empirical pdf of the t-statistics are obtained from 1000 samples of size corresponding to the available data with iid Gaussian innovations, under the null hypothesis of unit root. Given these simulations results, we also report the associated $\mathrm{P}$-value of the t-statistics. We immediately remark that the null hypothesis of unit root cannot be rejected for four countries, i.e. Germany, France, Italy and Canada. For these countries, it appears a good agreement between the three statistics. For the three other countries (US, UK and Japan), this is not the case. For the US, the FADF and ADF statistics allow to reject the null hypothesis, contrary to the the AR-OLS statistics. For the UK, from the FADF and AR-OLS statistics, we reject the unit root hypothesis, but not with the ADF one. Finally, for Japan, the FADF and ADF statistics lead to the rejection of the null hypothesis, but the AR-OLS one allows to its acceptance for a critical level of $5 \%$ (see the P-values in table 2).

In order to clarify these empirical results, we compute the power functions associated to the values of the nuisance parameters specific to these countries. FADF, ADF and AR-OLS power curves correspond to the solid line, the dashdotted line and the dotted line, respectively. The power curves 


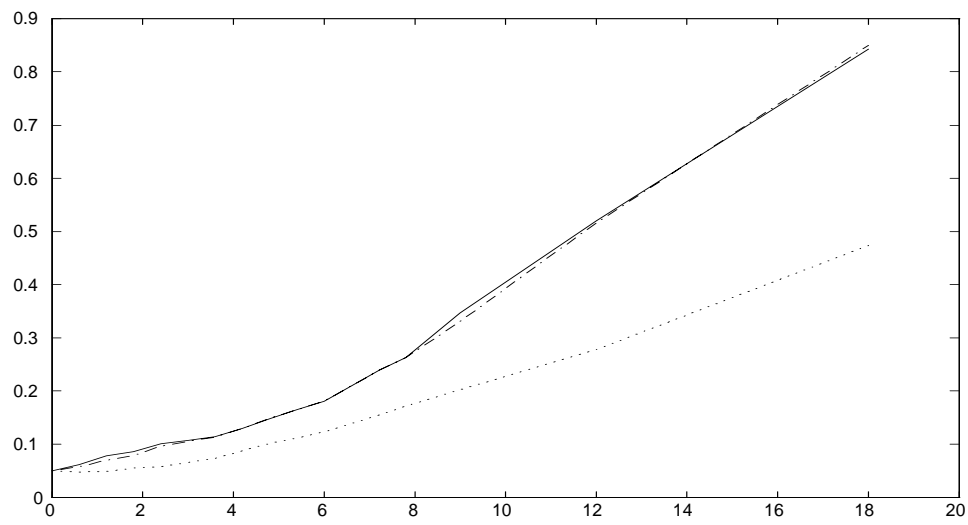

Figure 4: Power curves at 5\% of the t-statistics in the US case. FADF, ADF and AR-OLS power curves correspond to the solid line, the dashdotted line and the dotted line, respectively.

are obtained from 1000 samples of size corresponding to the data with iid Gaussian innovations. Each figure reports the power in terms of the parameter $c=T(1-\rho)$. For example, $c=6$ corresponds $^{13}$ to the value $\rho=0.9$

Figure 4 reports the power curves at $5 \%$ of the $t$-statistics in the US case, using the estimated values of the nuisance parameters from the covariance matrix of the residuals $(\eta=0.61, \tau=0.67)$. This figure shows that the AROLS t-statistics has less power than the FADF and ADF ones. This small sample experiment is thus in accordance with the previous asymptotics of Theorem 1. This result also indicates that the AR-OLS t-statistics leads to accept too often the null hypothesis of unit root.

Figure 5 reports the power curves at $5 \%$ of the $t$-statistics in the UK case, using the estimated values of $\eta=0.87$ and $\tau=0.39$. This figure shows that now the ADF t-statistics has less power than the FADF and AR-OLS ones. The UK case illustrates also the lack of power of ADF statistics.

\footnotetext{
${ }^{13} \mathrm{As}$ we focus on small samples properties of the statistics with $T$ fixed, it is equivalent to fix $c$ or to fix $\rho$ under the alternative.
} 


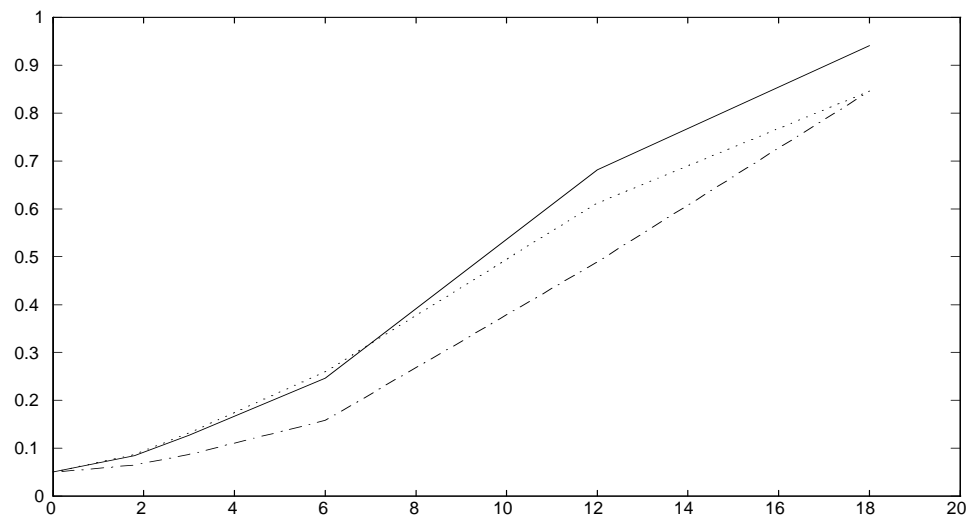

Figure 5: Power curves at 5\% of the t-statistics in the UK case. FADF, ADF and AR-OLS power curves correspond to the solid line, the dashdotted line and the dotted line, respectively.

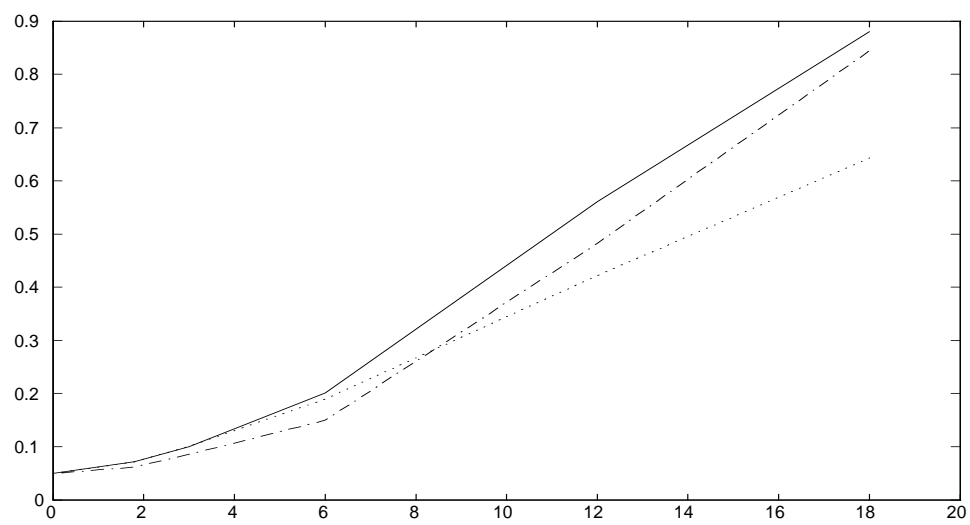

Figure 6: Power curves at 5\% of the t-statistics in the Japanese case. FADF, $\mathrm{ADF}$ and AR-OLS power curves correspond to the solid line, the dashdotted line and the dotted line, respectively. 
Finally, figure 6 reports the power curves at $5 \%$ of the $t$-statistics in the Japan case, with $\eta=0.85, \tau=0.60$. The FADF has better power properties than the ADF and AR-OLS ones. Moreover, for significant departures from unit root, the power of the $\mathrm{ADF}$ statistics is larger than the one of $\mathrm{AR}$ OLS statistics. The AR-OLS t-statistics allows to accept too often the null hypothesis of unit root.

These three simulations experiments show that the FADF statistics has globally better power properties than the two others. It seems that the use of this statistics may be recommended in order to prevent against any spurious conclusions on the stochastic properties of the effective public debt.

\section{Concluding remarks}

In this paper, we choose to test for sustainability of fiscal policy expressed as the stationarity of the debt to GDP ratio. This criterion appears more relevant for policy issues than the usual no-Ponzi game condition imposed on the discounted debt. Further, we introduce a new empirical strategy, building on a new test statistics, denoted FADF. This test statistics take advantages of the necessity of a feedback from inherited debt to current surplus as a joint condition for debt stationarity. The distribution of this statistics is shown to result from a mixture of $\mathrm{ADF}$ and normal ones and allows for potential power gains, even in small samples. Moreover, the limit distribution of the FADF statistics is compared to alternative statistics, associated to alternative univariate approaches to the stock-flow dynamics. By characterizing the limit distributions of the t-statistics obtained from the auxiliary equation considered in isolation, this paper also contributes to the study of inference on unbalanced regressions with potentially integrated regressors.

The empirical application performed on the G-7 countries illustrates the 
potential of this approach. Indeed, the FADF test allows to the rejection of the null hypothesis of unit root in the debt ratio for two more cases than the usual ADF test does. However, the non-sustainability of fiscal policy in continental European countries (and Canada) appears a robust empirical findings over the sample period considered in this study. This may provide a justification for a change in policy rules, as the one implemented under the auspice of Maastricht Treaty, so called convergence criteria. ${ }^{14}$

\footnotetext{
${ }^{14}$ See for instance, Artis and Marcellino (1998).
} 


\section{References}

[1] Artis, M. and Marcellino, M. (1998) "Fiscal Solvency and Fiscal Forcasting in Europe", CEPR Discussion papers, 1836, march

[2] Bohn, H.(1991) "On Testing the Sustainability of Budget Deficits in a Stochastic Environment", working paper no3, June, Center for Economic Studies. University of Munich.

[3] Buiter, W. and Patel, U. (1992) "Debt, Deficits and Inflation: an Application to the Public Finance of India" Journal of Public Economics, Vol 47, No 2 : 171-205.

[4] Campbell, J. and Perron, P. (1991) "Pitfalls and Opportunities: What Macroeconomists should Known About Unit Root", NBER Macroeconomics Annual, MIT Press.

[5] Cavanagh, C. , G. Elliott and Stock, J. (1995) "Inference in Models with Nearly Integrated Regressors", Econometric Theory, 11, 1131-1147.

[6] Elliott, G. and Stock, J. (1994)Inference in Time series Regression when the Order of Integration of a Regressor is Unknown" Econometric Theory, 10: $672-700$.

[7] Faruqee, H., Laxton, D. and Symansky, S. (1997) "Government Debt, Life-Cycle Income, and Liquidity Constraints: Beyond approximate Ricardian Equivalence" IMF Staff Papers, vol 44, No 3, september.

[8] Feve, P. and Henin, P.Y. (1996) "Assessing Sustainability of Public Deficits in a Stochastic Environment" , mimeo, CEPREMAP.

[9] Feve, P. , Hénin P.Y. and Jolivaldt P. (1997) "A Unit Root Test for Sustainability" mimeo CEPREMAP.

[10] Hakkio, G. and Rush, M. (1991) "Is the Budget Deficit too large?", Economic Inquiry, 29: 429-445.

[11] Hamilton, J. and Flavin, A. (1986) "On the Limitations of Government Borrowing: A Framework for Empirical Testing", The American Economic Review, 76 (4): 808-819, September.

[12] Hansen, B. (1995) "Rethinking the Univariate Approach to Unit Root Testing: using Covariates to Increase Power", Econometric Theory, 11: 1148-1171. 
[13] Holzmann, R., Herve, Y. and Demmel, R. (1996) "The Maastricht Fiscal Criteria: Required but Ineffective" Empirica, 25-51.

[14] Kremers, J. "US Federal Indebtness and the Conduct of Fiscal Policy" Journal of Monetary Economics, 23: 219-238.

[15] Phillips P. (1987a) "Toward an Unified Asymptotic Theory for Autoregression" Biometrika, $74: 535-547$.

[16] Phillips P. (1987b) "Time Series Regression with a Unit Root" Econometrica, 55 :277-301.

[17] Phillips P. and Hansen B.(1990) "Statistical inference in instrumental variables regression with I(1) processes" Review of Economic Studies, $57: 99-125$.

[18] Quintos, C. (1995) "Sustainability of the Deficit Process with Structural Shifts", Journal of Business Economic \&f Statistics, 13(4): 409-417, October.

[19] Schwert, G. (1989) "Test for Unit Root in Time Series Regression", Journal of Business Economics \& Statistics,7: 147-159.

[20] Trehan, B. and Walsh, C. (1988) "Common Trends, the Government Budget Constraint and Revenue Smoothing", Journal of Economic Dynamics and Control, 12:425-444,June/September.

[21] Trehan, B. and Walsh, C. (1991) "Testing Intertemporal Bubget Constraint: Theory and Applications to US Federal Budget and Current Account Deficit", Journal of Money Credit and Banking, 23 (2): 206223, May.

[22] Wickens M. and Uctum M. (1993) "The sustainability of current account deficits: a test of the US intertemporal budget constraint" Journal of Economic Dynamics and Control, 17(3):423-442, May.

[23] Wilcox, D. (1989) "The Sustainability of Government Deficits: Implications of the Present-Value Borrowing Constraint", Journal of Money Credit and Banking, 21 (3):291-306, August 


\section{Appendix}

To prove Theorem 1, we first use the following properties. The covariance matrix $\Sigma_{2}$ of $\left\{\varepsilon_{t}-u_{t}, u_{t}\right\}$ can be written as $\Sigma_{2}=P P^{\prime}$, using a Choleski decomposition, where the matrix $P$ is given by

$$
P=\sigma_{u}\left(\begin{array}{cc}
\left(1+\eta^{2}-2 \eta \tau\right)^{1 / 2} & 0 \\
\frac{\eta \tau-1}{\left(1+\eta^{2}-2 \eta \tau\right)^{1 / 2}} & \frac{\eta\left(1-\tau^{2}\right)^{1 / 2}}{\left(1+\eta^{2}-2 \eta \tau\right)^{1 / 2}}
\end{array}\right)
$$

Using this decomposition, we have under the local alternative $c \neq 0$

$$
\begin{aligned}
\frac{1}{T} \sum \bar{y}_{t-1}\left(\varepsilon_{t}-u_{t}\right) & \Longrightarrow \sigma_{u}^{2}\left(1+\eta^{2}-2 \eta \tau\right) \int_{0}^{1} B_{1}^{c *} d B_{1}(r) \\
\frac{1}{T^{2}} \sum \bar{y}_{t-1}^{2} & \Longrightarrow \sigma_{u}^{2}\left(1+\eta^{2}-2 \eta \tau\right) \int_{0}^{1}\left\{B_{1}^{c *}\right\}^{2} d r \\
\frac{1}{T} \sum \bar{y}_{t-1} u_{t} & \Longrightarrow \sigma_{u}^{2}(\eta \tau-1) \int_{0}^{1} B_{1}^{c *} d B_{1}(r)+\sigma_{u}^{2} \eta\left(1-\tau^{2}\right)^{1 / 2} \int_{0}^{1} B_{1}^{c *} d B_{2}(r)
\end{aligned}
$$

where $B_{2}$ is independent of $B_{1}^{*}$ and $B_{1}^{c *}$ and $\bar{y}_{t-1}=y_{t-1}-(1 / T) \sum y_{t-1}$. The functionals are $B_{1}^{c *}=B_{1}^{c}-\int_{0}^{1} B_{1}^{c}(s) d s$ and $B_{1}^{*}=B_{1}-\int_{0}^{1} B_{1}(s) d s$ (see Phillips (1987b)). Given these properties, we turns now to the limit distributions of the t-statistics.

The ML estimates of $\delta$ and $\alpha$ are given by $\widehat{\delta}=(1 / T) \sum\left(\Delta y_{t}+x_{t}\right)$ and $\widehat{\alpha}=(1 / T) \sum\left(x_{t}-\left(1-\widehat{\rho}_{F A D F}\right) y_{t-1}\right)$. Plugging the previous estimates into the score function associated to $\rho$ yields

$$
\widehat{\rho}_{F A D F}-1=\frac{\widehat{\tau}}{\widehat{\eta}}\left(\widehat{\rho}_{A D F}-1\right)+\left(1-\frac{\widehat{\tau}}{\widehat{\eta}}\right)\left(\widehat{\rho}_{A R-O L S}-1\right)
$$

where $\widehat{\rho}_{A D F}-1$ and $\widehat{\rho}_{A R-O L S}-1$ correspond to the single regression estimates, i.e. $\widehat{\rho}_{A D F}-1=\left(\sum \Delta \bar{y}_{t} \bar{y}_{t-1}\right)\left(\sum \bar{y}_{t-1}^{2}\right)^{-1}$ and $\widehat{\rho}_{A R-O L S}-1=$ $-\left(\sum \bar{x}_{t} \bar{y}_{t-1}\right)\left(\sum \bar{y}_{t-1}^{2}\right)^{-1}$ where $\Delta \bar{y}_{t}=\Delta y_{t}-(1 / T) \sum \Delta y_{t}, \bar{y}_{t-1}=y_{t-1}-$ $(1 / T) \sum y_{t-1}$ and $\bar{x}_{t}=x_{t}-(1 / T) \sum x_{t}$ The ML estimate of $\rho$ corresponds to a weighted sum of two single OLS estimates, with weights given by the estimated values of $\eta$ and $\tau$

$$
\frac{\widehat{\tau}}{\widehat{\eta}}=\frac{\frac{1}{T} \sum \Delta y_{t} x_{t}+\frac{1}{T} \sum x_{t}^{2}-\left(1-\widehat{\rho}_{F A D F}\right) \frac{1}{T} \sum\left(\Delta y_{t} y_{t-1}+x_{t} y_{t-1}\right)}{\frac{1}{T} \sum \widehat{\varepsilon}_{t}^{2}}
$$


Under the local alternative $c \neq 0$, as $T \rightarrow \infty, \frac{1}{T} \sum \Delta y_{t} x_{t}, \frac{1}{T} \sum x_{t}^{2}$ and $\frac{1}{T} \sum \widehat{\varepsilon}_{t}^{2}$ are consistent estimates of $\sigma_{u}^{2}(\eta \tau-1), \sigma_{u}^{2}$ and $\sigma_{\varepsilon}^{2}$, respectively. Thus, we have

$$
\frac{\widehat{\tau}}{\widehat{\eta}}=\frac{\tau}{\eta}+\left(\widehat{\rho}_{F A D F}-1\right) \frac{1}{\sigma_{\varepsilon}^{2}} \frac{1}{T} \sum\left(\Delta \bar{y}_{t} \bar{y}_{t-1}+\bar{x}_{t} \bar{y}_{t-1}\right)
$$

Plugging this expression into the the ML estimate $\widehat{\rho}_{F A D F}$ of $\rho$, one gets

$$
\widehat{\rho}_{F A D F}-1=\left\{\frac{\tau}{\eta}\left(\widehat{\rho}_{A D F}-1\right)+\left(1-\frac{\tau}{\eta}\right)\left(\widehat{\rho}_{A R-O L S}-1\right)\right\} \widehat{A}^{-1}
$$

where $\widehat{A}=1-\sigma_{\varepsilon}^{-2}\left(\frac{1}{T} \sum\left(\Delta \bar{y}_{t} \bar{y}_{t-1}+\bar{x}_{t} \bar{y}_{t-1}\right)\right)\left(\widehat{\rho}_{A D F}-\widehat{\rho}_{A R-O L S}\right)$. Under the local alternative, $T(\widehat{A}-1)$ admits the following asymptotics

$$
T(\widehat{A}-1) \Rightarrow \frac{\left\{(\eta-\tau) \int_{0}^{1} B_{1}^{c *} d B_{1}(r)+\left(1-\tau^{2}\right)^{1 / 2} \int_{0}^{1} B_{1}^{c *} d B_{2}(r)\right\}^{2}}{\left(1+\eta^{2}-2 \eta \tau\right) \int_{0}^{1}\left\{B_{1}^{c *}\right\}^{2} d r}
$$

$\widehat{A}$ is superconsistent and $\widehat{A} \rightarrow 1$. The statistics $T(\widehat{\rho}-1)$ is now given by

$$
T\left(\widehat{\rho}_{F A D F}-1\right)=\frac{\tau}{\eta} T\left(\widehat{\rho}_{A D F}-1\right)+\left(1-\frac{\tau}{\eta}\right) T\left(\widehat{\rho}_{A R-O L S}-1\right)
$$

where the limit distributions of the statistics associated to the two OLS estimates of $\rho$ are

$$
\begin{aligned}
T\left(\widehat{\rho}_{A D F}-1\right) & \Rightarrow-c+\frac{\int_{0}^{1} B_{1}^{c *}(r) d B_{1}}{\int_{0}^{1}\left\{B_{1}^{c *}(r)\right\}^{2} d r} \\
T\left(\widehat{\rho}_{A R-O L S}-1\right) & \Rightarrow-c+\frac{1-\eta \tau}{1+\eta^{2}-2 \eta \tau} \frac{\int_{0}^{1} B_{1}^{c *}(r) d B_{1}}{\int_{0}^{1}\left\{B_{1}^{c *}(r)\right\}^{2} d r}+\frac{\eta\left(1-\tau^{2}\right)^{1 / 2}}{1+\eta^{2}-2 \eta \tau} \frac{\int_{0}^{1} B_{1}^{c *}(r) d B_{2}}{\int_{0}^{1}\left\{B_{1}^{c *}(r)\right\}^{2} d r}
\end{aligned}
$$

One deduces the limit distribution of the statistics $T\left(\widehat{\rho}_{F A D F}-1\right)$

$$
T\left(\widehat{\rho}_{F A D F}-1\right) \Rightarrow-c+\xi^{2} \frac{\int_{0}^{1} B_{1}^{c *}(r) d B_{1}}{\int_{0}^{1}\left\{B_{1}^{c *}(r)\right\}^{2} d r}+\xi\left(1-\xi^{2}\right)^{1 / 2} \frac{\int_{0}^{1} B_{1}^{c *}(r) d B_{2}}{\int_{0}^{1}\left\{B_{1}^{c *}(r)\right\}^{2} d r}
$$

where $\xi=\left(1-\tau^{2}\right)^{1 / 2}\left(1+\eta^{2}-2 \eta \tau\right)^{-1 / 2}$. The ML standard error for the estimated value of $\rho$ is $\operatorname{se}\left(\widehat{\rho}_{F A D F}\right)=\left\{\sum \bar{y}_{t-1}^{2}\right\}^{-1 / 2}\left(1-\widehat{\tau}^{2}\right)^{1 / 2} \widehat{\sigma}_{u}$, where $\widehat{\sigma}_{u}^{2}$ denotes the estimate of the residuals variance $\widehat{\sigma}_{u}^{2}=(1 / T) \sum\left(\bar{x}_{t}-\left(1-\widehat{\rho}_{F A D F}\right) \bar{y}_{t-1}\right)^{2}$ 
The consistency of $\widehat{\rho}_{F A D F}$ implies $\widehat{\sigma}_{u}^{2} \rightarrow \sigma_{u}^{2}$ and $\widehat{\tau} \rightarrow \tau$. We thus deduce the following asymptotic

$$
\operatorname{Tse}\left(\widehat{\rho}_{F A D F}\right) \Rightarrow \xi\left\{\int_{0}^{1}\left\{B_{1}^{c *}(r)\right\}^{2} d r\right\}^{-1 / 2}
$$

Then, from the continuous mapping theorem, one obtains

$$
\begin{aligned}
t_{(F A D F)}^{\mu} \Rightarrow & -\frac{c}{\xi}\left\{\int_{0}^{1}\left\{B_{1}^{c}(r)\right\}^{2} d r\right\}^{1 / 2}+\xi \frac{\int_{0}^{1} B_{1}^{c}(r) d B_{1}}{\left\{\int_{0}^{1}\left\{B_{1}^{c}(r)\right\}^{2} d r\right\}^{1 / 2}} \\
& +\left(1-\xi^{2}\right)^{1 / 2} \frac{\int_{0}^{1} B_{1}^{c}(r) d B_{2}}{\left\{\int_{0}^{1}\left\{B_{1}^{c}(r)\right\}^{2} d r\right\}^{1 / 2}}
\end{aligned}
$$

Since $B_{2}$ is independent of $B_{1}$ and $B_{1}^{c},\left\{\int_{0}^{1} B_{1}^{c}(r) d B_{2}\right\}\left\{\int_{0}^{1}\left\{B_{1}^{c}(r)\right\}^{2} d r\right\}^{1 / 2}$ is distributed $\mathcal{N}(0,1)$ and is independent of $B_{1}^{c}$. The limit distribution of $t_{(A D F)}^{\mu}$ and $t_{(A R-O L S)}^{\mu}$ can be directly deduced from the limit distributions of $T\left(\widehat{\rho}_{A D F}-1\right)$ and $T\left(\widehat{\rho}_{A R-O L S}-1\right)$ and the continuous mapping theorem. This completes the proof 